\title{
Weed Dynamics and Yield of Chickpea (Cicer arietinum L.) as Influenced by Pre and Post-Emergence Herbicides
}

\author{
Vijay Laxmi Yadav $^{1 *}$, U.N. Shukla ${ }^{1}$ and M.L. Mehriya ${ }^{2}$ \\ ${ }^{1}$ Department of Agronomy, College of Agriculture, Mandor, Jodhpur-342304, India \\ ${ }^{2}$ Department of Agronomy, ARS, Mandor, Jodhpur-342304 \\ *Corresponding author
}

\section{Keywords}

Chickpea,

Herbicides, Weed density and Yield

Article Info

Accepted:

17 June 2018

Available Online:

100 July 2018

\section{A B S T R A C T}

A field experiment entitled "Efficacy of pre and post-emergence herbicides on growth and yield of chickpea (Cicer arietinum L.)" was conducted at Agricultural Research Station, Mandor, Jodhpur during rabi season of 2016-17. Field experiment was laid out in randomized block design (RBD) with sixteen treatments and replicated thrice. Sixteen treatments were tested among that two doses of each herbicides i.e. pendimethalin $(0.40$ and $0.60 \mathrm{~kg}$ a.i./ha), oxyfluorfen (100 and $200 \mathrm{~g}$ a.i./ha), imazethapyr (40 and $60 \mathrm{~g}$ a.i./ha) with their combinations as pre and post-emergence including weedy check and weed free taken for computing WCE and WI, respectively. According to treatments, different doses of pendimethalin and oxyfluorfen were applied as pre-mergence (within 3 DAS), while imazethapyr was applied as post-emergence (20 DAS). Among treatments, sequential application of pre and post-emergence herbicides i.e. pendimethalin@ $0.60 \mathrm{~kg}$ a.i. ha ${ }^{-1}$ (PE) + imazethapyr @ $60 \mathrm{~g}$ a.i. ha ${ }^{-1}$ at $20 \mathrm{DAS}\left(\mathrm{W}_{12}\right)$ significantly reduced weed density of Chenopodium murale L., Chenopodium album L. and Rumex dentatus L. at 30, 60, 90 DAS and at harvest, that improved the seed yield of chickpea, but it was equally effective with treatment had received pendimethalin@ $0.60 \mathrm{~kg}$ a.i. ha ${ }^{-1}(\mathrm{PE})+$ imazethapyr @ $40 \mathrm{~g}$ a.i. ha ${ }^{-1}$ at $20 \mathrm{DAS}\left(\mathrm{W}_{11}\right)$ as pre and post-emergence combination. Although, weed free recorded higher seed yield and showed significant superiority over rest of the weed management treatments, but it was statistically at par with $\mathrm{W}_{12}$ and $\mathrm{W}_{11}$.

\section{Introduction}

Chickpea is the third most important pulse crop in the world after french bean and field peas. India alone has nearly 52.5 per cent of the world acreage and production of chickpea. It is grown on acreage of 8.84 million hectare and producing 8.32 million tonnes with productivity of $942 \mathrm{~kg} \mathrm{ha}^{-1}$ during 2016-17 in India. Among states, Madhya Pradesh shared around 40 per cent in total production followed by Uttar Pradesh and Rajasthan contributing only 16 and 14 per cent, respectively. In Rajasthan, chickpea is successfully cultivated in arid and semi-arid districts and occupied at second rank in respect of area (1.26 mha) with low productivity (725 kg ha ${ }^{-1}$ (Anonymous, 2016). Chickpea is short stature crop with slow initial growth and therefore, heavily infested with wide spectrum of weeds. The early emergence and fast growth of the weeds 
lead to severe crop-weed competition for light, moisture, nutrients and space, which culminates in heavy reduction in growth and yield of chickpea and lessens the profitability (Chopra et al., 2003). About 40-45\% reduction in yield of chickpea due to severe infestation of weeds is estimated. If proper control measures are not taken, then the loss in terms of yield may increase up to 75 per cent in chickpea (Chaudhary et al., 2005). The initial 60 days period considered to critical for weed crop competition in chickpea (Singh and Singh 2000), but continuously facing of the scarcity of labour and increase in labour cost, manual weed control has become a difficult task. Suitable herbicide for effective control of mixed weed flora is required for better adoption in chickpea. Introduction of herbicides has made it possible to control a wide spectrum of weeds in pulses effectively at a remunerative cost. Many research workers from the various parts of the country has been reported that the application of pendimethalin as pre-emergence at $1.0 \mathrm{~kg} \mathrm{ha}^{-1}$ (Singh and Jain, 2017) and oxyfluorfen (80 $\left.\mathrm{g} \mathrm{ha}^{-1}\right)$ as weed control treatment (Patel et al., 2006) provided effective control of annual broad leaved and grassy weeds in chickpea field at an early stages. However, later flushes of weeds can only be control by application of imazethapyr as post-emergence (Rathod et al., 2017). Keeping in view these facts, the present investigation was undertaken to test the performance of pre and post-emergence herbicides either alone or combination with other weed management practices for providing effective weed control in chickpea.

\section{Materials and Methods}

\section{Experimental site}

The experiment was conducted at Agricultural Research Station, Mandor, Jodhpur during rabi season of 2016-17. Geographically, it is located between $26^{\circ} 15^{\prime} \mathrm{N}$ to $26^{\circ} 45^{\prime}$ North latitude and $73^{\circ} 00^{\prime} \mathrm{E}$ to latitude $73^{\circ} 29^{\prime}$ East longitude at an altitude of 231 meter above mean sea level. The soil was loamy sand in texture, slightly alkaline in reaction $(\mathrm{pH} 8.2)$, low in organic carbon $(0.13 \%)$ and available nitrogen $\left(174 \mathrm{~kg} \mathrm{ha}^{-1}\right)$, whereas medium in phosphorus (22.2 $\mathrm{kg} \mathrm{P}_{2} \mathrm{O}_{5} \mathrm{ha}^{-1}$ ) and available potassium $\left(325 \mathrm{~kg} \mathrm{~K}_{2} \mathrm{O} \mathrm{ha}^{-1}\right)$. The mean daily maximum and minimum temperature fluctuated between 21.8 to $39.8^{0} \mathrm{C}$ and 8.8 to $22.9^{0} \mathrm{C}$, respectively during the crop growing season.

\section{Experimental treatments}

The experiment was laid out in randomized block design (RBD) with sixteen weed control treatments, viz., $\mathrm{W}_{1}$-Weedy Check, $\mathrm{W}_{2}$-Weed free, $\mathrm{W}_{3}$-Pendimethalin @ $0.40 \mathrm{~kg}$ a.i./ha (PE), W4-Pendimethalin @ $0.60 \mathrm{~kg}$ a.i./ha (PE), W $5^{-}$Oxyfluorfen @ $100 \mathrm{~g}$ a.i./ha (PE), $\mathrm{W}_{6}$-Oxyfluorfen@200 g a.i./ha (PE), W 7- $^{-}$ Imazethapyr @40 g a.i./ha at 20 DAS, W 8- $^{-}$ Imazethapyr @60 g a.i./ha at 20 DAS, W $9^{-}$ Pendimethalin @ $0.40 \mathrm{~kg}$ a.i./ha (PE) + imazethapyr@40 g a.i./ha at 20 DAS, W $10^{-}$ Pendimethalin @ $0.40 \mathrm{~kg}$ a.i./ha (PE) + imazethapyr@60 g a.i./ha at 20 DAS, W 11- $^{-}$ Pendimethalin @ $0.60 \mathrm{~kg}$ a.i./ha (PE) + imazethapyr @40 g a.i./ha at 20 DAS, W $12^{-}$ Pendimethalin @ $0.60 \mathrm{~kg}$ a.i./ha (PE) + imazethapyr@60 g a.i./ha at 20 DAS, W $13^{-}$ Oxyfluorfen @ $100 \mathrm{~g}$ a.i./ha (PE + imazethapyr@40 g a.i./ha at 20 DAS, W 14- $^{-}$ Oxyfluorfen @ 100 g a.i./ha (PE) + imazethapyr @60 g a.i./ha at 20 DAS, W ${ }_{15^{-}}$ Oxyfluorfen @ 200 g a.i./ha (PE) + imazethapyr@40 g a.i./ha at 20 DAS and $\mathrm{W}_{16}$-Oxyfluorfen @ $200 \mathrm{~g}$ a.i./ha (PE) + imazethapyr@60 g a.i./ha at 20 DAS. As per treatments, pre-emergence application of pendimethalin and oxyfluorfen were applied within three days of sowing, while postemergence application of imazethapyr was applied at 20 DAS. These herbicides were sprayed with knapsack sprayer using flat fan 
nozzle in about 600 litres of water per hectare. A basal dose of $20 \mathrm{~kg} \mathrm{~N}$ and $40 \mathrm{~kg} \mathrm{P}_{2} \mathrm{O}_{5} \mathrm{ha}^{-1}$ were drilled uniformly before sowing through urea and diammonium phosphate, respectively in individual plot at the depth of 7 to $8 \mathrm{~cm}$ below the seed. After harvesting of crop, cleaned seeds were weighed to record seed yield per plot and then it converted in $\mathrm{kg} / \mathrm{ha}$. All the data were statistically analyzed to draw a valid conclusion.

\section{Results and Discussion}

\section{Effect of weed management treatments on weed dynamics}

\section{Relative composition of different weeds}

Data pertaining to relative composition of different weed flora as affected by different weed management practices (Table 1). It was observed that higher relative distribution of Chenopodium murale L. was reported among three weed flora followed by Chenopodium album L. and Rumex dentatus L. under particular set of the treatments.

The relative density of Rumex dentatus L. was higher under pre and post-emergence combination of pendimethalin @ $0.60 \mathrm{~kg}$ a.i./ha (PE) + imazethapyr @ $60 \mathrm{~g}$ a.i./ha at 20 DAS ( $\left.\mathrm{W}_{12}\right)$, whereas Chenopodium murale L. was higher under weedy check.

While relative density of Chenopodium album L. was higher under post-emergence application of imazethapyr with its two doses i.e. 60 and $40 \mathrm{~g}$ a.i./ha at all growth stages of crops except 30 DAS, where it was maximum under oxyfluorfen @ $100 \mathrm{~g}$ a.i./ha (PE) + imazethapyr @ $40 \mathrm{~g}$ a.i./ha at 20 DAS. However, mean relative composition in terms of percentage may be more in context to respective weed flora under particular treatment, but their densities were recorded lower under $\mathrm{W}_{11}$ and $\mathrm{W}_{12}$.

\section{Density of Chenopodium murale (No. $\mathrm{m}^{-2}$ )}

Chenopodium murale was one of the dominant weeds of the experimental plot (Table 2). The data revealed that density of Chenopodium murale significantly increased as the advancement of crop up to 60 DAS and there after decreased. It was found that combined application of pre and post-emergence herbicides i.e. pendimethalin @ $0.60 \mathrm{~kg}$ a.i./ha (PE)+imazethapyr @60 g a.i./ha at 20 DAS $\left(\mathrm{W}_{12}\right)$ recorded significantly lower weed density of Chenopodium murale, but it showed statistically at par with the treatment had received pendimethalin@0.60 kg a.i./ha (PE)+imazethapyr@40 g a.i./ha at 20 DAS $\left(\mathrm{W}_{11}\right)$ at all growth stages of crop. Due to season long weeding under weed free treatment $\left(\mathrm{W}_{2}\right)$ recorded none of the weed count and showed equally effective as $\mathrm{W}_{12}$ and $\mathrm{W}_{11}$ treatments. Similar results were reported by Kalyani (2011). Among alone application of herbicides, pre-emergence application of oxyfluorfen (100 and $200 \mathrm{~g}$ a.i. ha ${ }^{-1}$ ) proved inferior in this regard, whereas weedy check recorded higher density of Chenopodium murale at all growth stages of crop during experimentation. It was also found that when all herbicides integrated with imazethapyr reduced the density of Chenopodium murale compared to their sole application. Although, application of higher doses of pendimethalin, oxyfluorfen and imazethapyr found significantly superior over its lower doses (Dewangan et al., 2016).

\section{Chenopodium album (No. $\mathrm{m}^{-2}$ )}

Chenopodium album was the dominant weed of the experimental plot next to Chenopodium murale (Table 3). Scanning of data on density of Chenopodium album revealed significant reduction in its population by different weed management treatments. 
Table.1 Relative density of Chenopodium album, Chenopodium murale and Rumex dentatus as influenced various by weed management treatments

\begin{tabular}{|c|c|c|c|c|c|c|c|c|c|c|c|c|}
\hline \multirow{2}{*}{\multicolumn{2}{|c|}{ Treatments }} & \multicolumn{11}{|c|}{ Relative composition of weeds (\%) } \\
\hline & \multicolumn{3}{|c|}{30 DAS } & \multicolumn{3}{|c|}{60 DAS } & \multicolumn{3}{|c|}{90 DAS } & \multicolumn{3}{|c|}{ At harvest } \\
\hline & $\mathbf{A}$ & B & $\mathbf{C}$ & $\mathbf{A}$ & B & $\mathbf{C}$ & $\mathbf{A}$ & B & $\mathbf{C}$ & $\mathbf{A}$ & B & $\mathbf{C}$ \\
\hline $\mathbf{W}_{1}$ & 20.38 & 75.79 & 3.83 & 17.89 & 77.85 & 4.26 & 23.86 & 71.72 & 4.42 & 23.16 & 72.88 & 3.95 \\
\hline $\mathbf{W}_{2}$ & 0.00 & 0.00 & 0.00 & 0.00 & 0.00 & 0.00 & 0.00 & 0.00 & 0.00 & 0.00 & 0.00 & 0.00 \\
\hline $\mathbf{W}_{3}$ & 27.81 & 59.76 & 12.43 & 26.60 & 59.61 & 13.79 & 27.66 & 57.45 & 14.89 & 25.81 & 61.29 & 12.90 \\
\hline $\mathbf{W}_{4}$ & 25.66 & 61.18 & 13.16 & 26.67 & 58.33 & 15.00 & 28.13 & 56.25 & 15.63 & 25.93 & 60.49 & 13.58 \\
\hline $\mathbf{W}_{5}$ & 24.28 & 67.34 & 8.38 & 24.93 & 65.42 & 9.65 & 25.09 & 67.01 & 7.90 & 22.86 & 69.05 & 8.10 \\
\hline$W_{6}$ & 23.91 & 67.39 & 8.70 & 24.44 & 65.73 & 9.83 & 25.09 & 66.67 & 8.24 & 23.32 & 68.39 & 8.29 \\
\hline $\mathbf{W}_{7}$ & 27.45 & 60.78 & 11.76 & 28.92 & 58.63 & 12.45 & 28.49 & 61.29 & 10.22 & 27.07 & 61.65 & 11.28 \\
\hline $\mathbf{W}_{8}$ & 27.57 & 60.00 & 12.43 & 28.70 & 58.26 & 13.04 & 28.74 & 60.48 & 10.78 & 28.21 & 60.68 & 11.11 \\
\hline $\mathbf{W}_{9}$ & 24.59 & 54.10 & 21.31 & 20.69 & 50.00 & 29.31 & 20.45 & 54.55 & 25.00 & 25.93 & 51.85 & 22.22 \\
\hline $\mathbf{W}_{10}$ & 23.08 & 53.85 & 23.08 & 20.00 & 50.00 & 30.00 & 18.92 & 54.05 & 27.03 & 23.81 & 52.38 & 23.81 \\
\hline $\mathbf{W}_{11}$ & 22.58 & 41.94 & 35.48 & 17.24 & 41.38 & 41.38 & 19.05 & 42.86 & 38.10 & 26.67 & 46.67 & 26.67 \\
\hline $\mathbf{W}_{12}$ & 20.83 & 37.50 & 41.67 & 16.67 & 37.50 & 45.83 & 13.33 & 40.00 & 46.67 & 20.00 & 50.00 & 30.00 \\
\hline $\mathbf{W}_{13}$ & 28.16 & 54.37 & 17.48 & 24.49 & 51.02 & 24.49 & 25.93 & 50.62 & 23.46 & 26.67 & 56.67 & 16.67 \\
\hline $\mathrm{W}_{14}$ & 27.17 & 54.35 & 18.48 & 23.60 & 50.56 & 25.84 & 26.39 & 50.00 & 23.61 & 27.45 & 54.90 & 17.65 \\
\hline$W_{15}$ & 25.00 & 53.95 & 21.05 & 23.29 & 46.58 & 30.14 & 24.56 & 47.37 & 28.07 & 27.50 & 52.50 & 20.00 \\
\hline $\mathbf{W}_{16}$ & 24.24 & 53.03 & 22.73 & 22.73 & 45.45 & 31.82 & 25.53 & 42.55 & 31.91 & 30.30 & 48.48 & 21.21 \\
\hline
\end{tabular}

A-Chenopodium album, B- Chenopodium murale, C- Rumex dentatus 
Table.2 Density of Chenopodium murale as influenced by various weed management treatments

\begin{tabular}{|c|c|c|c|c|}
\hline \multirow[t]{2}{*}{ Treatments } & \multicolumn{4}{|c|}{ Chenopodium murale $\left(\right.$ No. $\left.\mathrm{m}^{-2}\right)$} \\
\hline & 30 DAS & 60 DAS & 90 DAS & At Harvest \\
\hline $\mathbf{W}_{1}$ & $14.81(224.33)$ & $16.67(280.00)$ & $12.67(162.33)$ & $11.33(129.00)$ \\
\hline $\mathbf{W}_{2}$ & $0.71(0.00)$ & $0.71(0.00)$ & $0.71(0.00)$ & $0.71(0.00)$ \\
\hline $\mathbf{W}_{\mathbf{3}}$ & $5.83(33.67)$ & $6.36(40.33)$ & $5.21(27.00)$ & $4.39(19.00)$ \\
\hline $\mathbf{W}_{4}$ & $5.60(31.00)$ & $5.93(35.00)$ & $4.91(24.00)$ & $4.07(16.33)$ \\
\hline $\mathbf{W}_{5}$ & $8.83(77.67)$ & $9.02(81.33)$ & $8.08(65.00)$ & $6.96(48.33)$ \\
\hline $\mathbf{W}_{6}$ & $8.53(72.33)$ & $8.84(78.00)$ & $7.73(59.33)$ & $6.66(44.00)$ \\
\hline $\mathbf{W}_{7}$ & $6.45(41.33)$ & $6.99(48.67)$ & $6.20(38.00)$ & $5.26(27.33)$ \\
\hline $\mathbf{W}_{8}$ & $6.10(37.00)$ & $6.70(44.67)$ & $5.84(33.67)$ & $4.90(23.67)$ \\
\hline $\mathbf{W}_{9}$ & 3.38 (11.00) & $3.17(9.67)$ & $2.88(8.00)$ & $2.26(4.67)$ \\
\hline $\mathbf{W}_{10}$ & $3.11(9.33)$ & $2.94(8.33)$ & $2.65(6.67)$ & $2.02(3.67)$ \\
\hline $\mathbf{W}_{11}$ & $2.18(4.33)$ & $2.11(4.00)$ & $1.86(3.00)$ & $1.68(2.33)$ \\
\hline $\mathrm{W}_{12}$ & $1.86(3.00)$ & $1.86(3.00)$ & $1.56(2.00)$ & $1.44(1.67)$ \\
\hline $\mathbf{W}_{13}$ & 4.37 (18.67) & 4.13 (16.67) & 3.74 (13.67) & 3.42 (11.33) \\
\hline$W_{14}$ & $4.13(16.67)$ & $3.93(15.00)$ & $3.52(12.00)$ & $3.12(9.33)$ \\
\hline $\mathrm{W}_{15}$ & 3.74 (13.67) & $3.41(11.33)$ & $3.05(9.00)$ & $2.72(7.00)$ \\
\hline $\mathbf{W}_{16}$ & 3.48 (11.67) & $3.24(10.00)$ & $2.67(6.67)$ & $2.40(5.33)$ \\
\hline SEm \pm & 0.24 & 0.21 & 0.19 & 0.16 \\
\hline C.D. $(P=0.05)$ & 0.71 & 0.61 & 0.55 & 0.47 \\
\hline Figures in parent & & & & \\
\hline
\end{tabular}




\begin{tabular}{|c|c|c|c|c|}
\hline \multirow{2}{*}{ Treatments } & \multicolumn{4}{|c|}{ Chenopodium album (No. $\left.\mathrm{m}^{-2}\right)$} \\
\hline & 30 DAS & 60 DAS & 90 DAS & At Harvest \\
\hline $\mathrm{W}_{1}$ & $7.78(60.33)$ & $8.03(64.33)$ & $7.38(54.00)$ & $6.44(41.00)$ \\
\hline $\mathrm{W}_{2}$ & $0.71(0.00)$ & $0.71(0.00)$ & $0.71(0.00)$ & $0.71(0.00)$ \\
\hline $\mathrm{W}_{3}$ & 3.99 (15.67) & $4.30(18.00)$ & $3.67(13.00)$ & $2.91(8.00)$ \\
\hline $\mathrm{W}_{4}$ & $3.65(13.00)$ & $4.04(16.00)$ & $3.53(12.00)$ & $2.72(7.00)$ \\
\hline $\mathrm{W}_{5}$ & $5.31(28.00)$ & $5.60(31.00)$ & $4.98(24.33)$ & $4.06(16.00)$ \\
\hline $\mathrm{W}_{6}$ & $5.09(25.67)$ & $5.42(29.00)$ & $4.77(22.33)$ & $3.94(15.00)$ \\
\hline $\mathrm{W}_{7}$ & $4.36(18.67)$ & $4.92(24.00)$ & $4.26(17.67)$ & $3.53(12.00)$ \\
\hline $\mathrm{W}_{8}$ & $4.16(17.00)$ & $4.73(22.00)$ & $4.06(16.00)$ & $3.39(11.00)$ \\
\hline $\mathrm{W}_{9}$ & $2.32(5.00)$ & $2.10(4.00)$ & $1.84(3.00)$ & $1.64(2.33)$ \\
\hline $\mathrm{W}_{10}$ & $2.11(4.00)$ & $1.93(3.33)$ & $1.64(2.33)$ & $1.44(1.67)$ \\
\hline $\mathrm{W}_{11}$ & $1.68(2.33)$ & $1.46(1.67)$ & $1.34(1.33)$ & $1.34(1.33)$ \\
\hline $\mathrm{W}_{12}$ & $1.46(1.67)$ & $1.34(1.33)$ & $1.05(0.67)$ & $1.05(0.67)$ \\
\hline $\mathrm{W}_{13}$ & $3.17(9.67)$ & $2.88(8.00)$ & $2.72(7.00)$ & $2.40(5.33)$ \\
\hline $\mathrm{W}_{14}$ & $2.95(8.33)$ & $2.72(7.00)$ & $2.59(6.33)$ & $2.26(4.67)$ \\
\hline $\mathrm{W}_{15}$ & $2.58(6.33)$ & $2.46(5.67)$ & $2.24(4.67)$ & $2.00(3.67)$ \\
\hline $\mathrm{W}_{16}$ & $2.40(5.33)$ & $2.33(5.00)$ & $2.08(4.00)$ & $1.93(3.33)$ \\
\hline SEm \pm & 0.14 & 0.12 & 0.10 & 0.08 \\
\hline C.D. $(P=0.05)$ & 0.40 & 0.35 & 0.29 & 0.23 \\
\hline
\end{tabular}


Table.4 Density of Rumex dentatus as influenced by various weed management treatments

\begin{tabular}{ccccc}
\hline Treatments & \multicolumn{4}{c}{ Rumex dentatus $\left(\right.$ No. $\mathbf{~ m}^{-2}$ ) } \\
\cline { 2 - 5 } & 30 DAS & 60 DAS & 90 DAS & At Harvest \\
\hline $\mathrm{W}_{1}$ & $3.44(11.33)$ & $3.97(15.33)$ & $3.21(10.00)$ & $2.72(7.00)$ \\
$\mathrm{W}_{2}$ & $0.71(0.00)$ & $0.71(0.00)$ & $0.71(0.00)$ & $0.71(0.00)$ \\
$\mathrm{W}_{3}$ & $2.72(7.00)$ & $3.10(9.33)$ & $2.70(7.00)$ & $2.08(4.00)$ \\
$\mathrm{W}_{4}$ & $2.66(6.67)$ & $3.06(9.00)$ & $2.66(6.67)$ & $2.02(3.67)$ \\
$\mathrm{W}_{5}$ & $3.17(9.67)$ & $3.52(12.00)$ & $2.82(7.67)$ & $2.46(5.67)$ \\
$\mathrm{W}_{6}$ & $3.12(9.33)$ & $3.48(11.67)$ & $2.79(7.33)$ & $2.40(5.33)$ \\
$\mathrm{W}_{7}$ & $2.88(8.00)$ & $3.28(10.33)$ & $2.60(6.33)$ & $2.32(5.00)$ \\
$\mathrm{W}_{8}$ & $2.84(7.67)$ & $3.22(10.00)$ & $2.53(6.00)$ & $2.18(4.33)$ \\
$\mathrm{W}_{9}$ & $2.16(4.33)$ & $2.46(5.67)$ & $2.02(3.67)$ & $1.56(2.00)$ \\
$\mathrm{W}_{10}$ & $2.10(4.00)$ & $2.32(5.00)$ & $1.93(3.33)$ & $1.46(1.67)$ \\
$\mathrm{W}_{11}$ & $2.02(3.67)$ & $2.08(4.00)$ & $1.76(2.67)$ & $1.34(1.33)$ \\
$\mathrm{W}_{12}$ & $1.93(3.33)$ & $2.02(3.67)$ & $1.68(2.33)$ & $1.22(1.00)$ \\
$\mathrm{W}_{13}$ & $2.5(6.00)$ & $2.88(8.00)$ & $2.58(6.33)$ & $1.93(3.33)$ \\
$\mathrm{W}_{14}$ & $2.46(5.67)$ & $2.83(7.67)$ & $2.46(5.67)$ & $1.86(3.00)$ \\
$\mathrm{W}_{15}$ & $2.39(5.33)$ & $2.79(7.33)$ & $2.39(5.33)$ & $1.74(2.67)$ \\
$\mathrm{W}_{16}$ & $2.30(5.00)$ & $2.70(7.00)$ & $2.32(5.00)$ & $1.66(2.33)$ \\
$\mathrm{SEm} \pm$ & 0.08 & 0.09 & 0.07 & 0.05 \\
C.D. $(P=0.05)$ & 0.23 & 0.26 & 0.20 & 0.14 \\
\hline F
\end{tabular}

Figures in parentheses are the original value 
Table.5 Seed yield of chickpea as influenced by various weed management

\begin{tabular}{|c|c|}
\hline Treatments & $\begin{array}{c}\text { Seed } \\
\text { yield } \\
\left(\mathrm{kg} \mathrm{ha}^{-1}\right)\end{array}$ \\
\hline $\mathrm{W}_{1} \quad$ Weedy & 728.33 \\
\hline $\mathrm{W}_{2} \quad$ Weed free (Season long) & 2327.33 \\
\hline $\mathrm{W}_{3} \quad$ Pendimethalin @ $0.40 \mathrm{~kg}$ a.i. ha ${ }^{-1}$ (PE) & 1561.33 \\
\hline Pendimethalin@0.60kg a.i. ha ${ }^{-1}(\mathrm{PE})$ & 1607.33 \\
\hline $\mathrm{W}_{5} \quad$ Oxyflourfen @100 g a.i. ha ${ }^{-1}(\mathrm{PE})$ & 1189.00 \\
\hline $\mathrm{W}_{6} \quad$ Oxyflourfen @ $200 \mathrm{~g}$ a.i. ha ${ }^{-1}(\mathrm{PE})$ & 1274.67 \\
\hline Imazethapyr@40g a.i. ha ${ }^{-1}$ at 20 DAS & 1398.67 \\
\hline $\mathrm{W}_{8} \quad$ Imazethapyr @60 g a.i. ha ${ }^{-1}$ at $20 \mathrm{DAS}$ & 1489.00 \\
\hline $\begin{array}{l}\text { W9 Pendimethalin @ } 0.40 \mathrm{~kg} \text { a.i. ha }{ }^{-1} \text { (PE) + Imazethapyr @ } 40 \mathrm{~g} \text { a.i. } \\
\text { ha }^{-1} \text { at } 20 \text { DAS }\end{array}$ & 1991.33 \\
\hline 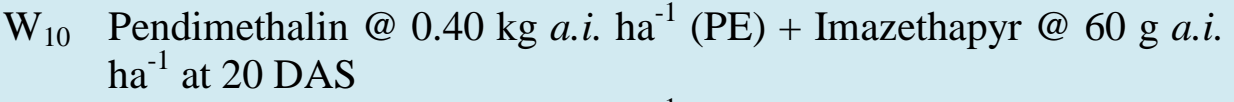 & 2077.67 \\
\hline $\begin{array}{l}\mathrm{W}_{11} \begin{array}{l}\text { Pendimethalin @ } 0.60 \mathrm{~kg} \text { a.i. ha } \\
\text { ha }^{-1} \text { at } 20 \mathrm{DAS}\end{array}\end{array}$ & 2231.33 \\
\hline $\begin{array}{l}\mathrm{W}_{12} \begin{array}{l}\text { Pendimethalin @ } 0.60 \mathrm{~kg} \text { a.i. ha } \\
\text { ha }^{-1} \text { at } 20 \mathrm{DAS}\end{array}\end{array}$ & 2303.33 \\
\hline $\begin{array}{l}\mathrm{W}_{13} \text { Oxyflourfen @100 g a.i. ha }{ }^{-1}(\mathrm{PE})+\text { Imazethapyr @ } 40 \mathrm{~g} \text { a.i. ha }{ }^{-1} \\
\text { at } 20 \text { DAS }\end{array}$ & 1715.33 \\
\hline $\begin{array}{l}\mathrm{W}_{14} \text { Oxyflourfen @100 g a.i. ha }{ }^{-1}(\mathrm{PE})+\text { Imazethapyr @ } 60 \mathrm{~g} \text { a.i. ha }{ }^{-1} \\
\text { at } 20 \text { DAS }\end{array}$ & 1749.67 \\
\hline $\begin{array}{l}\mathrm{W}_{15} \text { Oxyflourfen@200 g a.i. ha }{ }^{-1}(\mathrm{PE})+\text { Imazethapyr @ } 40 \mathrm{~g} \text { a.i. } \mathrm{ha}^{-1} \\
\text { at } 20 \mathrm{DAS}\end{array}$ & 1855.33 \\
\hline $\begin{array}{l}\mathrm{W}_{16} \text { Oxyflourfen @ } 200 \mathrm{~g} \text { a.i. ha }{ }^{-1}(\mathrm{PE})+\text { Imazethapyr @ } 60 \mathrm{~g} \text { a.i. ha }{ }^{-1} \\
\text { at } 20 \text { DAS }\end{array}$ & 1872.67 \\
\hline SEm \pm & 36.91 \\
\hline $\mathrm{CD}(P=0.05)$ & 104.23 \\
\hline
\end{tabular}


It was noticed that combined application of pre and post-emergence herbicides i.e. pendimethalin @ $0.60 \mathrm{~kg}$ a.i./ha (PE) + imazethapyr@60 g a.i./ha at 20 DAS (W 12 ) had significantly lowered density of Chenopodium album and showed its superiority over rest of the treatments including weedy check at all growth stages of crop. However, this treatment $\left(\mathrm{W}_{12}\right)$ found at par with pendimethalin @ $0.60 \mathrm{~kg}$ a.i./ha (PE) + imazethapyr @40 g a.i./ha at 20 DAS $\left(\mathrm{W}_{11}\right)$. These results are in the conformity with the work of Singh et al., (2014). Among individual categories of herbicides and their doses, application of oxyfluorfen (100 and $200 \mathrm{~g}$ a.i. $\mathrm{ha}^{-1}$ ) as pre-emergence showed poor in controlling weed population at all growth stages.

\section{Rumex dentatus (No. $\mathrm{m}^{-2}$ )}

Rumex dentatus was the third major weed flora in the experimental plot (Table 4). A cursory glance of data indicated that sequential application of pre and postemergence herbicides i.e. pendimethalin @ $0.60 \mathrm{~kg}$ a.i./ha (PE) + imazethapyr @ $60 \mathrm{~g}$ a.i./ha at 20 DAS $\left(\mathrm{W}_{12}\right)$ produced marked variation in the density of Rumex dentatus and recorded significantly minimum density that established its superiority over other treatments at all growth stages, though it remained at par with pre and post-emergence application of herbicides i.e. pendimethalin @ $0.60 \mathrm{~kg}$ a.i./ha (PE) + imazethapyr @ $40 \mathrm{~g}$ a.i./ha at 20 DAS $\left(\mathrm{W}_{11}\right)$. Similar results were reported by Kalyani (2011) and Poonia and Pithia (2013). Weedy check $\left(\mathrm{W}_{1}\right)$ heavily infested with weed flora of Rumex dentatus and recorded more in density as compared to other weed management treatments during field trial. Similarly, other treatments also recorded minimum density of the same weed in contrast to weedy check during the year of experimentation.
Effect of weed management treatments on yield of chickpea

Seed yield is an important parameter which decides the efficiency and superiority of a particular treatment over other treatments. Sequential application of pre and postemergence herbicide i.e. pendimethalin @ $0.60 \mathrm{~kg}$ a.i./ha (PE) + imazethapyr @60 g a.i./ha at 20 DAS $\left(\mathrm{W}_{12}\right)$ and pendimethalin @ $0.60 \mathrm{~kg}$ a.i./ha (PE) + imazethapyr @ $40 \mathrm{~g}$ a.i./ha at 20 DAS $\left(\mathrm{W}_{11}\right)$ were recorded significantly higher seed yield (2303.33 and $2231.33 \mathrm{~kg} \mathrm{ha}^{-1}$ ), respectively over other weed management treatments, but these were at par with each other and also showed equally effective as weed free treatment $\left(\mathrm{W}_{2}\right)$. While lesser difference of increments between $\mathrm{W}_{2}, \mathrm{~W}_{12}$ and $\mathrm{W}_{11}$ treatments were noticed due to similar weed control across the crop growth period. It was might be due to lesser infestation of weeds that encourage proper translocation of photosynthesis from source to sink. Such condition may increase the seed production ratio in total produce. Similar results were also reported by Dubey et al., (2018) and Kalyani (2011) (Table 5).

It is concluded that combined application of pre and post-emergence herbicides i.e. pendimethalin @ $0.60 \mathrm{~kg}$ a.i./ha (PE) + imazethapyr @ 60 g a.i./ha at 20 DAS recorded lower density of weeds viz., Chenopodium murale, Chenopodium album and Rumex dentatus and also recorded higher seed yield, but equally effective with similar combinations and lower doses herbicide i.e. pendimethalin @ $0.60 \mathrm{~kg}$ a.i./ha (PE) + imazethapyr@40 g a.i./ha at 20 DAS in this regards. Imazethapyr as post-emergence caused plant injury, but recover faster rate at later stages. Keeping in all views in mind, pendimethalin @ $0.60 \mathrm{~kg}$ a.i./ha (PE) + imazethapyr@40 g a.i./ha at 20 DAS as pre and post-emergence herbicide may be feasible and taken for further research. 


\section{References}

nonymous. (2016). Agricultural Statistics at a Glance-2015. Directorate of Economics and Statistics, Department of Agriculture, Co-operation and Farmers Welfare, Ministry of Agriculture and Farmers Welfare, Govt. of India, New Delhi.

Chaudhary, B. M., Patel, J. J., and Delvadia, D. R. (2005). Effect of weed management practices and seed rates on weeds and yield of chickpea. Indian Journal of Weed Sciences. 37(3\&4):271-272.

Chopra, N., Chopra, N. K., and Singh, H. P. (2003). Loss in seed yield and quality due to weed stress in chickpea (Cicer arietinum L.). Indian Journal of Agricultural Sciences. 73(6):350-351.

Dewangan, M., Singh, A. P., Choudhary, T., Diproshan, and Kumar, B. (2016). Management of complex weed flora in chickpea. Indian Journal of Weed Science. 48(1):79-82.

Dubey, S. K., Kumar, A., Singh, D., Pratap, T., and Chaurasiya, A. (2018). Effect of different weed control measures on performance of chickpea under irrigated condition. International Journal of Current Microbiology and Applied Sciences. 7(5): 3103-3111.

Kalyani, D. (2011). Integrated weed management in chickpea (Cicer arietinum L.). Thesis submitted to ANGRAU, Rajendranagar,
Hyderabad.

Patel, B. D., Patel, V. J., Patel, J. B., and Patel, R. B. (2006). Effect of fertilizers and weed management practices on weed control in chickpea (Cicer arietinum L.) under middle Gujrat conditions. Indian Journal of crop Sciences. 1:180-183.

Poonia, T. C., and Pithia, M. S. (2013). Pre and post-emergence herbicides for weed management in chickpea. Indian Journal of Weed Science. 45(3):223225.

Rathod, P. S., Patil, D. H., and Dodamani, B. M. (2017). Evaluation of time and dose of imazethapyr in controlling weeds of chickpea (Cicer arietinum L.). Legume Research. 40(5):906-910.

Singh, A., and Jain, N. (2017). Integrated weed management in chickpea. Indian Journal of Weed Science, 49(1): 9394.

Singh , S., and Singh A., N. (2000). Cropweed competition in chickpea: Challenges and strategies for the New Millenium. In National Symposium on Agronomy, p 199. 15-18 November 2000. GAU Campus, Junagarh.

Singh, R. P., Verma, S. K., Singh, R. K., and Idnani, L. K. (2014). Influence of sowing dates and weed management on weed growth and nutrients depletion by weeds and uptake by chickpea (Cicer arietinum) under rainfed condition. Indian Journal of Agricultural Sciences. 84(4):468-72.

\section{How to cite this article:}

Vijay Laxmi Yadav, U.N. Shukla and Mehriya, M.L. 2018. Weed Dynamics and Yield of Chickpea (Cicer arietinum L.) as Influenced by Pre and Post-Emergence Herbicides Int.J.Curr.Microbiol.App.Sci. 7(07): 2523-2532. doi: https://doi.org/10.20546/ijcmas.2018.707.296 